\title{
Butyric Acid and Lactic Acid in Aqueous Solutions as Solubilizers for Carcinogenic Hydrocarbons
}

\author{
PER EKW ALL and LARS SJ ÖBLOM
}

Institute of Physical Chemistry, Abo Akademi, Abo, Finland

Rury and coworkers ${ }^{1,2}$ have shown by measuring the specific volumes and B freezing point lowerings that an association leading to micelle formation takes place in aqueous solutions of $n$-butyric acid. As in soap solutions micelles begin to form at a definite concentration, "the critical concentration for micelle formation", which in this case is about $1.1 \mathrm{M}$ at $35^{\circ} \mathrm{C}$. Since soaps and other association colloids are able to solubilize water-insoluble carcinogenic hydrocarbons $^{3-5}$, it was of interest to investigate whether these hydrocarbons are solubilized also by the butyric acid micelles. Owing to the fact that butyric acid has a very short carbon chain, it would be expected that also the micelles it forms are quite small and hence have a poor solubilizing power.

$n$-Butyric acid solutions of increasing concentration were shaken 48 hours at $40^{\circ} \mathrm{C}$ with an excess of finely powdered 3,4-benzpyrene (BP). In pure water and in dilute butyric acid solution the polycyclic hydrocarbon is practically insoluble, but when the butyric acid concentration attains a certain value the solubility begins to increase as shown by the fluorescence in ultraviolet light (Fig. 1, curve 1). Already in a $0.85 M$ butyric acid solution an extremly weak fluorescence is noted, which becomes gradually stronger with increasing butyric acid concentration. A marked fluorescence is first evident in the $1.08 M$ solution and above 1.35 $M$ the intensity of the fluorescence increases rapidly. This latter values agrees well with the value for the critical concentration which is evident from Bury's data ${ }^{1,2}$. It is clear that the actual micelle formation begins at this concentration, but it may be that in the presence of benzpyrene micelle formation is preceded by an association of lesser degree which begins at a concentration near $0.85 M$.

The behaviour is much the same in butyric acid solutions containing hydrochloric acid or sodium chloride $(0.1 N)$, or both $(0.1 N+0.1 N)$. The abrupt 

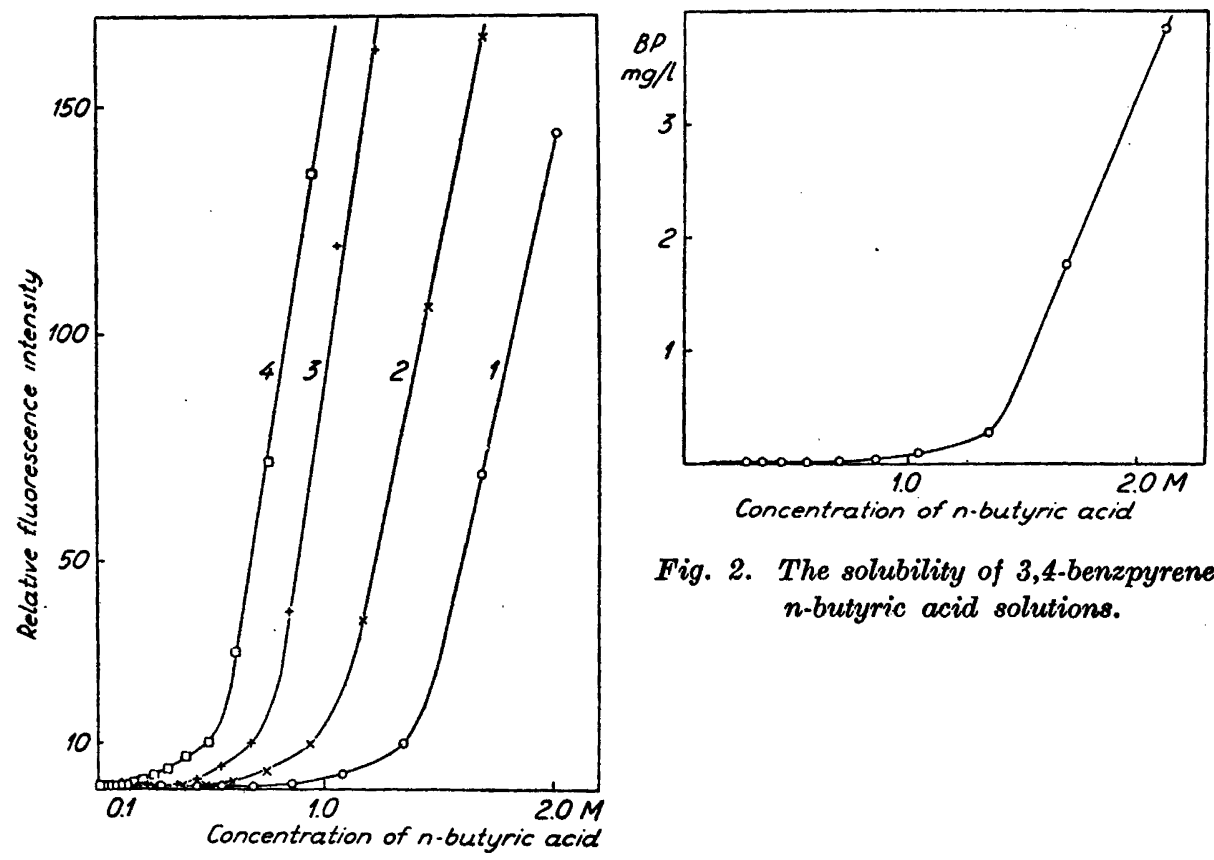

Fig. 2. The solubility of 3,4-benzpyrene in n-butyric acid solutions.

Fig. 1. The fluorescence intensity curves of solutions saturated with 3,4-benzpyrene. Curve 1. n-Butyric acid alone.

- 2. n-Butyric acid and d,l-lactic acid, molar ratio unity.

- 3. n-Butyric acid and d,l-lactic acid molar ratio two.

- 4. n-Butyric acid, d,l-lactic acid and sodium taurocholate, molar ratios 1 : $2: 0.19$.

rise in the fluorescence occurs in all these cases when the butyric acid concentration is approximately $1.1 M$; the first weak fluorescence is observed at. a somewhat lower concentration (about $0.7 M$ ) in the presence of the electrolytes.

The solubility of benzpyrene in a $1.24 M$ butyric acid solution was found to be $0.18 \mathrm{mg}$ per litre. With this value as a basis we have estimated from the fluorescence intensities the solubility of benzpyrene at the other butyric acid concentrations (Fig. 2). The values obtained in this manner are only approximate, but will serve for orientation. In the $2 M$ solution the solubility has increased to about $2.7 \mathrm{mg}$ benzpyrene per litre.

Several other fatty acids were studied to determine whether their aqueous solution are also able to solubilize benzpyrene. Saturated solutions of caproic, caprylic, nonylic, capric and oleic acids were prepared and saturated with benzpyrene in the manner described above. No fluorescence, however, was 
observed in any of these solutions which shows that no benzpyrene was solubilized. This is probably due to the fact that the solubilities of these acids in water are lower than their respective critical concentrations.

In the literature we have not found any data which would indicate that lactic acid undergoes micelle formation. Despite this we have studied whether aqueous solutions of this acid are able to effect solution of benzpyrene. The results were positive. A marked fluorescence was observed in $d, l$-lactic acid solutions saturated with benzpyrene beginning with a lactic acid concentration of about 4.3 $M$ (Fig. 3). The fluorescence increased rapidly above this concentration. A weak fluorescence is noted when the lactic acid concentration is about 2.8 M. The measurements were to some extent rendered difficult by the weak greenish fluorescence of the pure lactic acid solutions. An approximate determination of the solubility of benzpyrene based on fluorescence measurements gave a value of about $2.3 \mathrm{mg}$ per litre of $6.8 M$ lactic acid (38 weight per cent lactic acid). - As long as micelle formation by lactic acid has not been proved by other methods, and since very high concentrations of the acid are involved, we prefer to attribute the solubility of benzpyrene to a hydrotropic effect on the part of lactic acid.

The ability of butyric acid to solubilize benzpyrene is greatly increased in the presence of lactic acid. When the molar ratio of lactic acid to butyric acid is unity the fluorescence begins to increase rapidly when the butyric acid concentration exceeds $0.85 M$; when the molar ratio is two the fluorescence increases already when the butyric acid concentration exceeds $0.65 M$. A weak fluorescence is observed in these cases at butyric acid concentrations of $\mathbf{0 . 5}$ and $0.3 M$ respectively. In none of these solutions was the butyric acid concentration high enough for it alone to be able to solubilize benzpyrene. Thus it appears that mixed micelle formation is involved, $i . e$, butyric acid micelles containing lactic acid molecules are formed.

The ability of butyric and lactic acids to solubilize carcinogenic hydrocarbons is of biological interest since in certain conditions these acids are found free in the stomach. When the hydrochloric acid content of the stomach is low, lactic acid fermentation can take place in the stomach and according to the literature this is often followed by butyric acid fermentation ${ }^{6}$. On the other hand, if under extreme conditions (lack of $\mathrm{HCl}$ ) the lipase of the stomach is able to split fatty substances, it is the lower fatty acids in the glycerides that will be liberated first 7; it is possible that this may lead to the formation of some free butyric acid in the stomach. Furthermore, appreciable amounts of free lactic acid enter into the stomach with food (sour whole milk, bread). In this connection it may also be noted that lactic acid is produced by oxidative metabolism in the cells of the gastric mucosa ${ }^{12}$. 


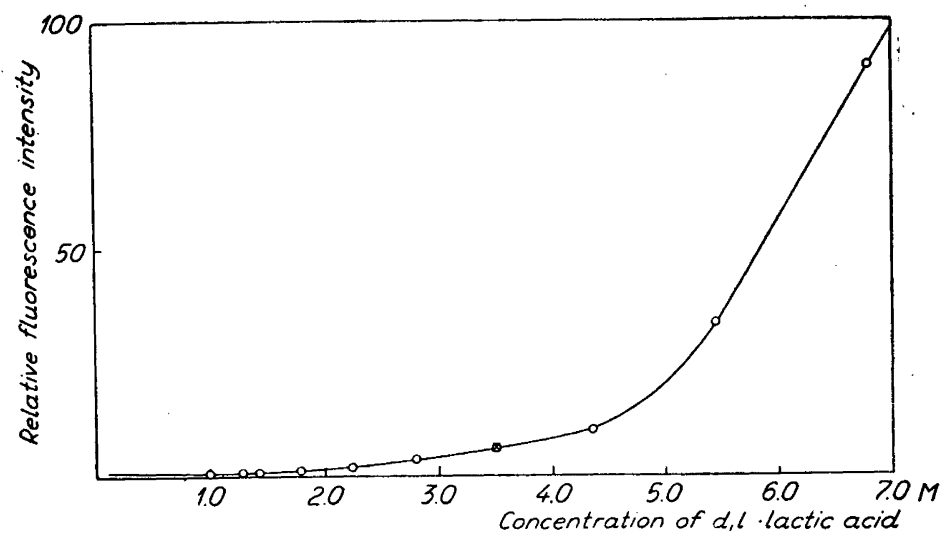

Fig. 3. The fluorescence intensities of d,l-lactic acid solutions saturated with 3,4-benzpyrene.

The investigations of Setälä, Ekwall and their coworkers ${ }^{5,8,10}$ have shown that benzpyrene solubilized by association colloids is able to penetrate into the glandular tissue of the stomach in mice and cats despite the presence of the protective mucous layer of the stomach. It therefore appears probable that also butyric acid solutions are able to induce a penetration of carcinogens solubilized in them.

It has previously been pointed out $5,8,11$ that the bile is an association colloid solution consisting primarily of bile acid and fatty acid salts, lecithin and cholesterol, which is able to solubilize carcinogens and that this property is not lost even in acid solutions ( $\mathrm{pH} 1)$. When bile enters the stomach, the conditions become such that carcinogenic hydrocarbons can penetrate into the tissues of the stomach wall $5,8,11$.

The present investigation thus shows that in the stomach in addition to bile there can occur also other association colloid systems capable of effecting the solubilization of carcinogenic hydrocarbons, viz. solutions of butyric and lactic acids. It is of interest to know how these micelle systems affect each other's ability to solubilize carcinogenic hydrocarbons. With taurocholate present in the ratio of one mole to 70 moles butyric acid a definite solubilization is noted already in an aqueous solution that is $0.3 M$ in butyric acid (a weak fluorescence begins at a concentration of $0.2 M$ ). In these solutions the critical concentration of taurocholate has not yet been exceeded. On the other hand, the critical concentration of taurocholate is lowered when butyric and lactic acids are present in concentrations that are alone insufficient to effect the solubilization of benzpyrene. Thus, when bile enters the stomach when the latter contains butyric and lactic acids the conditions 
for the solubilization of carcinogenic hydrocarbons and hence for their penetration into the walls of the stomach are improved.

Since, in order to have any effect, the carcinogen must first penetrate into the tissues, our investigations suggest a new way by which gastric cancer may be effected by chemical carcinogenesis. This possibility possesses considerable interest since statistics show that in humans gastric cancers and low stomach acidities are frequently encountered together.

It is proposed to continue the investigations to determine the power of butyric acid - lactic acid solutions and of butyric acid - lactic acid - bile solutions to promote the penetration of carcinogens into the glandular tissue of the stomachs of animals, to determine the amounts of butyric and lactic acids present in the human stomach in different conditions and to study the ability of the stomach content to solubilize carcinogenic hydrocarbons.

\section{SUMMARY}

Aqueous solutions of $n$-butyric acid of concentrations above $1.1 M$ solubilize the fluorescent carcinogenic hydrocarbon 3,4-benzpyrene. Benzpyrene dissolves also in aqueous solutions of $d, l$-lactic acid when the concentration of the acid exceeds $4.3 M$.

In the presence of lactic acid the ability of butyric acid to solubilize benzpyrene is increased greatly. Sodium taurocholate in concentrations below its critical concentration has the same effect.

The results are discussed from the point of view of chemical carcinogenesis in the stomach.

A grant from the Sigrid Juselius' Stiftelse is gratefully_acknowledged.

\section{REFERENCES}

1. Jones, E. R., and Bury, Ch. R. Phil. Mag. (7) 4 (1927) 841.

2. Bury, Ch. R., and Grindley, J. J. Chem. Soc. (1929) 679.

3. Ekwall, P. VI Nordiska Kemistmötet, Lund 25-29 augusti 1947 (1948). Lund.

4. Ekwall, P., and Setälä, K. Acta Chem. Scand. 2 (1948) 733.

5. Ekwall, P., Setälä, K., and Sjöblom, L. Acta Chem. Scand. 5 (1951) 175.

6. Boas, I. Diagnostik und Therapie der Magenkrankheiten (1925) 164-165. Leipzig.

7. Peltola, E. Über die Zusammensetzung und enzymatische Spaltung des Kuhmilchfettes, Dissertation, Jokioinen (1944).

8. Ekwall, P., and Setälä, K. Acta Intern. Union against Cancer 7 (1950) 120.

9. Ermala, P., Setälä, K., and Ekwall, P. Cancer Research. 11 (1951) 753.

10. Ekwall, P., Ermala, P., Setälä, K., and Sjöblom, L. Cancer Research. 11 (1951) 758.

11. Ekwall, P., Lindström, E., and Setälä, K. Acta Chem. Scand. 5 (1951) 990.

12. Davenport, H. W. Gastroenterology 9 (1947) 293. 